\title{
ARTIGO 28 Original
}

\section{TRATAMENTO DA TUBERCULOSE NA ESTRATÉGIA SAÚDE DA FAMÍLIA: OLHAR DO PROFISSIONAL}

Shirley Ribeiro dos Santos Linhares Elisabete Pimenta Araújo Paz²
ORCID: http://lattes.cnpq.br/9510859002114575
ORCID: https://orcid.org/0000-0002-1692-0253

Objetivos: compreender o cotidiano assistencial dos profissionais de saúde referentes aos cuidados ofertados durante o tratamento da tuberculose. Método: Pesquisa fenomenológica, realizada com 52 profissionais de saúde em unidades de Saúde da Família do município do Rio de Janeiro. Os dados foram obtidos por meio de entrevistas semiestruturadas e analisadas a luz da hermenêutica heideggeriana. Resultados: A análise revelou que no encontro assistencial reside a possibilidade dos profissionais de saúde ultrapassarem o modo de ser indiferente dos serviços, para oferecerem um cuidado autêntico que reconheça o outro no difícil transcurso da doença para a cura. Conclusão: Faz-se necessário conciliar o conhecimento técnico-científico que objetiva o sucesso do tratamento da tuberculose com as dimensões subjetivas dos que apresentam a doença, pois reside no diálogo com o outro a possibilidade de superar as dificuldades comuns de quem cuida e de quem precisa restabelecer a saúde e o ritmo da vida.

Descritores: Tuberculose, Pesquisa qualitativa, Estratégia Saúde da Família.

Objectives: to understand daily care of health professionals regarding the care offered during the treatment of tuberculosis. Method: Phenomenological research, carried out with 52 health professionals at Family Health Units in the city of Rio de Janeiro. Data were obtained through semi-structured interviews and analyzed in light of Heideggerian hermeneutics. Results: The analysis revealed that in the care encounter, it is possible for health professionals to go beyond the indifferent way of services, to offer an authentic care that recognizes the other in the difficult course of the illness for the cure. Conclusion: It is necessary to reconcile the technical-scientific knowledge that aims at the success of the treatment of tuberculosis with the subjective dimensions of those who present the disease, since it resides in the dialogue with the other the possibility of overcoming the common difficulties of those who care for and who needs to restore health and the pace of life.

Descriptors: Tuberculosis, Qualitative research, Family Health Strategy.

Objetivos: comprender el cotidiano asistencial de los profesionales de salud referentes a los cuidados ofrecidos durante el tratamiento de la tuberculosis. Método: Investigación fenomenológica, realizada con 52 profesionales de salud en unidades de Salud de la Familia del municipio Río de Janeiro. Los datos fueron obtenidos por medio de entrevistas semiestructuradas y analizadas a la luz de la hermenéutica heideggeriana. Resultados: El análisis reveló que en el encuentro asistencial reside la posibilidad de los profesionales de salud sobrepasar el modo de ser indiferente de los servicios, para ofrecer un cuidado auténtico que reconozca al otro en el difícil transcurso de la enfermedad para la curación. Conclusión: Se hace necesario conciliar el conocimiento técnico científico que objetiva el éxito del tratamiento de la tuberculosis con las dimensiones subjetivas de los que presentan la enfermedad, pues reside en el diálogo con el otro la posibilidad de superar las dificultades comunes de quien cuida y de quien es necesario restablecer la salud y el ritmo de la vida.

Descriptores: Tuberculosis, Investigación cualitativa, Estrategia Salud de la Familia; 


\section{INTRODUÇÃO}

A história natural da tuberculose (TB) comprova que os avanços tecnológicos relacionados à terapia medicamentosa e à descoberta de medidas preventivas, ocorridos principalmente no século XX, proporcionaram importantes mudanças no tratamento e representação social da doença $a^{(1)}$. Porém, ao contrário dos avanços obtidos, a tuberculose ainda se destaca como uma das doenças que mais mata no mundo, principalmente em países subdesenvolvidos. Sua ocorrência encontra-se diretamente relacionada ao modo como se organizam os processos de produção e organização social, assim como à implementação de políticas de controle da doença ${ }^{(1-3)}$.

Apesar dos avanços conseguidos no combate a esta doença, o Brasil é um dos 48 países priorizados pela Organização Mundial de Saúde (OMS) para o combate à tuberculose. Atualmente o país ocupa a 20 a posição em relação à carga da doença e a 19ạ no que se refere à coinfecção TB-HIV, com um total de 69.569 casos novos da doença notificados em 2017, apresentando uma taxa de incidência de 33,5 casos $/ 100$ mil hab(4.5).

Nesse cenário, para avançar no controle da doença, a estratégia utilizada para seu combate não pode ser apenas a descentralização das ações para a Atenção Primária à Saúde (APS). Faz-se necessário a reestruturação da rede assistencial que garanta o diagnóstico precoce e a realização da supervisão do tratamento (impactando na redução das taxas de abandono), aliada ao combate à miséria ${ }^{(6)}$.

No Brasil, paraalém do aumento da cobertura da Estratégia Saúde da Família (ESF) como uma política estruturante do sistema de saúde, há a necessidade de qualificação dos serviços de atenção primária à saúde como porta de entrada para o acolhimento e acompanhamento do paciente, pois a não adesão amplia o tempo e o custo de tratamento, interferindo negativamente no desfecho esperado que é a cura da doença ${ }^{(7-10,14)}$

Reconhece-se que para o efetivo controle da doença é necessário que o doente tenha sido diagnosticado em tempo oportuno e realize o tratamento com esquema terapêutico adequado, em doses corretas e no tempo adequado. As consultas de acompanhamento clínico devem ser mensais, com a adoção da prática de educação em saúde e da estratégia de tratamento supervisionado (DOTS), para isso, é fundamental que profissionais e políticas de saúde transcendam o caráter fragmentado e reducionista das ações $^{(11-13)}$

Assim, torna-se imprescindivel que os profissionais de saúde reconheçam o processo de adoecer por TB como um fenômeno multidimensional e se disponham a prestar uma assistência individualizada, não se restringindo apenas aos aspectos clínicos e entendendo o doente de tuberculose como um ser único e com peculiaridades físicas, psíquicas e sociais, uma vez que cada individuo vivencia de modo particular o momento do adoecer e tratar ${ }^{(7-10)}$.

Considerando a situação epidemiológica da TB e a importância dos serviços de saúde no seu controle, visando o alcance das metas estabelecidas pela OMS que impactam na coletividade, este estudo tem como objetivo compreender o cotidiano assistencial e as experiências dos profissionais de saúde referentes aos cuidados ofertados durante o tratamento da tuberculose.

\section{MÉTODO}

Pesquisa qualitativa de abordagem fenomenológica com analítica sustentada no referencial de Martin Heidegger(15-16). A opção por este referencial emergiu da necessidade de conhecer qual o significado que o profissional de saúde é capaz de dar às experiências que vive durante o atendimento ao portador de tuberculose no cotidiano assistencial pois estes significados influenciam seu modo de prestar assistência ${ }^{(15-17)}$

O cenário de estudo foram cinco unidades de Atenção Primária, situadas na área da Leopoldina Norte, no município do Rio de Janeiro. Os participantes foram 25 médicos e 27 enfermeiros. O critério de inclusão foi possuir experiência mínima de um ano no atendimento ao portador de tuberculose. Foram excluídos aqueles que se encontravam afastados do trabalho por motivo de férias, licença ou outra razão laboral no período da obtenção dos dados.

A coleta de dados ocorreu com a realização de entrevistas no período de dezembro de 2014 a março de 2015 . exclusivamente nas dependências das unidades de saúde, em dias e horários agendados para não prejudicar as rotinas de atendimento. Utilizou-se roteiro semiestruturado de questões que abordaram a experiência do atendimento ao portador de tuberculose. As entrevistas foram registradas com o auxílio de um gravador MP3 após o consentimento dos entrevistados e imediatamente transcritas. Para garantir o anonimato dos participantes, entrevistas foram codificadas pela letra P de profissionais, seguidas pelo número de ordem de realização das mesmas. A coleta dos dados foi interrompida após todos serem entrevistados.

A análise das entrevistas fundamentadas no pensar de Martin Heidegger se deu em dois momentos. Iniciou-se com leituras atentivas dos depoimentos em busca dos significados expressos pelos profissionais. A redução fenomenológica permitiu a distinção das estruturas essenciais em detrimento das ocasionais, privilegiando as primeiras, uma vez que responderam ao objetivo da investigação. Essas estruturas essenciais constituíram unidades de significação que trouxeram a compreensão ôntica do fenômeno, ou seja, a compreensão imediata daquilo que foi interrogado, o senso 
comum $^{(17)}$

No segundo momento metódico, a hermenêutica, buscouse a essência contida nos depoimentos por meio de novas leituras baseadas no pensamento filosófico de Heidegger para desvelar o fenômeno, que não se mostra diretamente no fato, mas nele está velado, apontando a necessidade de desconstrução do factual para fazer emergir as estruturas essenciais do discurso ${ }^{(17)}$.

A pesquisa foi aprovada pelo Comitê de Ética em Pesquisa da Escola de Enfermagem Anna Nery-Universidade Federal do Rio de Janeiro e Comitê de Ética em Pesquisa da Secretaria Municipal de Saúde do Rio de Janeiro recebendo parecer favorável.

\section{RESULTADOS}

Os discursos revelaram as experiências dos profissionais ao realizarem atendimento aos pacientes portadores de tuberculose nas unidades de APS, o que deu origem a três unidades de significados apresentadas a seguir.

\section{A influência do discurso normativo em um cotidiano impessoal}

Esta unidade mostra que os profissionais de saúde enfatizam as ações assistenciais preconizadas pelo Programa de Controle da Tuberculose. Valorizam as orientações técnicas sobre o tratamento, pois estão preocupados em evitar a disseminação da doença na comunidade.

Falo sempre que se tomar o remédio direito, não deixar de tomar a medicação, se alimentar e tratar adequadamente [...] que eles vão ficar curados. É uma doença que tem cura e que mata muito menos atualmente se seguir o tratamento corretamente $(P I)$

A gente orienta toda a questão da transmissão, a importância de seguir o tratamento correto até o final, o risco que todo familiar e que toda pessoa que tem contato com ele hoje se expõe se por acaso ele não conseguir finalizar este tratamento de forma correta (P31)

Os

$$
\text { profissionais }
$$

sabem que

tratamento não é simples de ser seguido, principalmente devido ao tempo prolongado e aos efeitos adversos da medicação, mas acreditam que mediante informações sobre a tuberculose, seu tratamento e efeitos colaterais, conseguirão manter a pessoa vinculada ao serviço, estabelecendo um bom caminho para a adesão ao tratamento.

Alguns não têm aquele comprometimento de estar todo dia fazendo uso da medicação. A maioria das vezes a dificuldade é quando o paciente não entende, tanto pelo médico quanto pelo enfermeiro, a gravidade, entendeu? (P4)

Desde o início eu oriento o paciente sobre o que é tuberculose, como se pega e sobre o tratamento. Falo sobre os efeitos colaterais dos remédios, da urina que fica vermelha e digo que é normal, para ele não parar de tomar o remédio que é assim mesmo (P27).

A gente orienta esse paciente que ele tem que fazer o DOTS, que ele tem que tomar corretamente a medicação, observar os efeitos colaterais dessa medicação, orientar sobre a cura da tuberculose, que se ele aderir ao tratamento diariamente ele vai ficar curado daquela doença (PII).

É importante para os profissionais que além de conhecer a doença, os doentes reconheçam que a adesão medicamentosa é essencial para vencê-la. Porém sabem que o portador possui dinâmicas próprias de vida que interferem no seguimento ambulatorial desejado.

\section{Caminhando para o reencontro do cuidado para com o} outro

Apesar da preocupação com a interrupção da cadeia de transmissão, mais do que tratar a tuberculose, os profissionais sabem que conhecer o usuário em suas particularidades devido ao estigma da doença é uma questão importante no projeto terapêutico

Este tipo de cuidado tem muita importância, pois são usuários que transmitem a doença para a comunidade. Por isso é importante que realizamos a busca ativa e o tratamento sem perder este usuário pelo caminho, por causa do preconceito (P43)

Para a clínica da familia é primordial que o atendimento seja bem realizado, pois é uma doença que tem muito tabu, as pessoas ignoram que têm e se têm não querem dizer. Então quando a gente consegue identificar, estamos evitando a disseminação da doença e diminuindo incidência. Para mim é muito gratificante detectar e cuidar, tirar o estigma da doença (P45)

Os profissionais demonstraram preocupação com as condições sociais e emocionais dos portadores de tuberculose e valorizaram o vínculo requerido nas práticas de saúde para o sucesso do tratamento. Reconhecem que com a manutenção do vínculo poderão estabelecer laços de confiança e cooperação com o usuário de modo a levá-lo a compreender a importância de seu tratamento e seu papel na quebra da cadeia de transmissão. 
Os que eu estava acompanhando eu tinha um vínculo, chegavam até mim, falavam abertamente dos problemas, sobre as drogas [...], porque que não tomou medicação naquele dia, porque que estava há tantos dias sem tomar, os problemas com a família e [...]isso ajudava bastante ter uma relação mais aberta com a gente (P3)

[...]como todo mundo precisa de uma atenção, eles também precisam de uma atenção. Acho que até maior porque geralmente são pessoas mais vulneráveis, que precisam de sentir acolhidas, não é só chegar aqui e pegar o remédio e vai e tomar o remédio em casa (P6)

Mais do que tratar a tuberculose, os profissionais sabem que precisam considerar os significados sociais advindos do adoecer, pois ela afeta não apenas o vigor físico, mas também o emocional e a dinâmica familiar dos que estão diagnosticados como portadores de TB.

\section{Preocupando-se com as dificuldades no cotidiano assistencial}

Esta categoria apresenta as dificuldades vivenciadas pelos profissionais para manterem o usuário em tratamento até a cura. Existe a possibilidade de que com toda orientação fornecida, a pessoa não queira continuar tomando a medicação seja pela melhora dos sintomas logo, ou pelos efeitos adversos que afetam o organismo já debilitado. Mesmo conhecendo todo o desconforto causado pelo medicamento, continuar incentivando ou apoiando os usuários na tomada da medicação é parte de seu ofício assistencial.

Na minha opinião é um dos pacientes mais difíceis de você conseguir manter ele certinho até o final[...] é um paciente que já está numa situação de maior vulnerabilidade, pode estar associado ao álcool, ou de uso de drogas, ou que mora numa casa com muita gente e aí as vezes não é bem-uindo naquela casa por estar com tuberculose. (P22)

As dificuldades são inúmeras. É a violência desse lugar, que não deixa a gente se programar para fazer uma $V D$, nós nunca sabemos quando vai dar tiro. É o usuário que não entende que o tratamento dura seis meses e que ele não pode parar de tomar o remédio só porque melhorou, é a dificuldade de conseguir um CPF para fazer o cadastro do usuário na unidade (P48)

Muitas vezes com as comunidades subjugadas a uma dinâmica de violência pela guerra do tráfico, com tiroteios a qualquer hora, não se pode garantir que a realização do TDO. Estas parecem ser dificuldades incontornáveis, acima da capacidade de resposta dos profissionais e que não favorecem o sucesso que esperam obter ao prestarem assistência a pessoas com tuberculose.

\section{DISCUSSÃO:}

O sofrimento é que desencadeia a busca de assistência e, para o doente, é algo palpável, que pode incapacitá-lo e que oscila conforme a autoculpabilização e, sobretudo, da estigmatização social. Os profissionais de saúde sabem o quanto a família e os amigos são essenciais na vida dos pacientes, já que são os recursos disponiveis da rede de apoio social que podem thes oferecer auxilio e amparo(14,18,19)

O homem, ser-aí em seu cotidiano relaciona-se com outros seres de um modo predominante, o qual ele chama de impessoal. Sob o domínio do impessoal os profissionais lançam mão de todos os recursos de comunicação para que o doente entenda, absorva e cumpra o programa de tratamento, ajudando-o a superar o inesperado do adoecimento. Ao seguir esta dinâmica, o profissional de saúde pode apresentar um comportamento inautêntico, desviando-se de sua incumbência essencial, ou seja, ser um ser do cuidado (17,20).

Na cotidianidade ôntica, habitual, dos serviços de saúde os profissionais tornam-se menos atento aos temores, às preocupações e às necessidades dos pacientes. Assumem a responsabilidade de cuidar cumprindo protocolos terapêuticos, pois tecnicamente este é o único caminho que podem oferecer. Outros estudos também comprovam esses achados, uma vez que os profissionais valorizam primariamente as orientações técnicas necessárias para que o paciente prossiga tomando seus medicamentos $(9,10,20)$.

A não resolutividade dos profissionais frente às necessidades do doente pode resultar em não adesão ao tratamento. Devido à importância da adesão terapêutica para o sucesso do tratamento e para o controle da doença, diversos estudos sobre o abandono do tratamento da TB têm sido realizados no Brasil. Dentre as causas mais frequentes, foram identificadas: informações deficitárias sobre a doença, organização do serviço de saúde e intolerância medicamentosa ${ }^{(9,21,22)}$

Mesmo na limitação ôntica que faz com que os profissionais se relacionem sob o domínio do impessoal, na primazia da tradição medicalizante é possivel o estabelecimento de vínculo decorrente da supervisão terapêutica, entre os profissionais e usuários. Investigações anteriores apontaram o vínculo desenvolvido durante o tratamento como um fator importante para garantia e continuidade do tratamento, pois se manifesta em atitudes baseadas no cuidado, na atenção particularizada, na escuta e confiança, fazendo-os se sentir seguros e participativos no cuidado a sua saúde ${ }^{(7,9114,22)}$.

A preocupação com o restabelecimento da saúde tanto 
por parte dos profissionais como por parte dos usuários com tuberculose coloca o ser diante da possibilidade própria de querer ver-se novamente em toda sua integralidade. Nesse sentido, o doente passa a reconhecer o valor e a dimensão do tratamento, aceitando o profissional de saúde como um aliado na busca da cura. A prática assistencial pautada na subjetividade revela o cuidado autêntico, que dá acesso ao mais íntimo desejo de cada indivíduo na retomada de sua saúde ${ }^{(20,21)}$

O cuidado centrado no usuário é uma possibilidade na ESF, desde que as práticas de saúde sejam voltadas, e, orientadas às suas necessidades de forma integral. Neste modelo de atenção, o estar-com-os-outros é favorecido pela proximidade com que a equipe permanece presente no cotidiano de vida das pessoas, promovendo um cuidado libertador(14,17,19).

Neste estudo, verificou-se que os profissionais podem manifestar dois modos de ser no cotidiano assistencial: um voltado para a ocupação, onde se deixa guiar pela desatenção às percepções e comportamentos dos pacientes em relação à doença e a cura. O outro voltado para a preocupação, no qual o profissional se importa e compreende a necessidade de se levar em consideração na dinâmica assistencial, o pensar, o sentir e o agir do doente, para além das questões biológicas, sendo então um ser-com-os-outros, abrindo possibilidades de um cuidado que restabeleça a confiança em si e na equipe de saúde ${ }^{(16,17)}$

Orientado por tal abertura, o cuidado ofertado se fortalece durante o tratamento, compartilhando com o doente as decisões sobre a sua saúde. Esta relação formada com o profissional permite a instauração de um modo de assistir que possibilita a realização mais própria da humanidade do homem, que dialoga e não domina, abrindo-se a possibilidade de transcender o cuidado fundado nos aspectos normativos, que é a tradição tecnicista da saúde ${ }^{(9,23)}$.

A produção de cuidados de saúde e de enfermagem deve buscar conhecer as concepções dos indivíduos sobre o seu processo de saúde-doença, o que se constitui eixo importante, quando se objetiva o êxito do processo terapêutico e o alcance de melhores níveis de qualidade de vida para os usuários dos serviços de saúde ${ }^{(24)}$
O estudo apresentou algumas limitações no que se refere à realidade do municipio do Rio de Janeiro, pois foram pesquisados dois bairros de uma mesma área de planejamento, o que dificulta a generalização dos achados para outros contextos sociais.

\section{CONSIDERAÇÕES FINAIS}

Entende-se que o processo de adoecimento do paciente com tuberculose está relacionado a múltiplos fatores, e não apenas aos efeitos quea doença causa no corpo. Os resultados mostraram que em alguns momentos os pacientes estão sujeitos a impessoalidade dos profissionais, que se equivocam ao presumir que o conhecimento técnico é garantia da cura.

O "estar doente" depende da percepção do pessoa em relação à sua condição. Sendo assim, para a garantia da cura é importante que o profissional tenha sensibilidade para ouvir o paciente, conhecer sua realidade e assim, estabelecer um cuidado pautado na compreensão do outro, ajudando-o em suas dificuldades e pactuando ações que permitam decisões factiveis de serem cumpridas no dia-a-dia. O cuidado autêntico não é tarefa simples e requer dos envolvidos no processo um olhar atento, às necessidades e possibilidades que cada doente pode assumir. Desta forma será possivel a construção de um projeto terapêutico balizado pela integralidade e solidariedade assistencial.

Conclui-se que no cuidado voltado à pessoas em tratamento de tuberculose a primazia do tratamento medicamentoso não deve se sobrepor a individualidade da pessoa acometida pela tuberculose, ainda que nos encontros do seguimento ambulatorial, as complexas rotinas das unidades de saúde os profissionais de saúde não permitam que todos sejam atendidos em suas singularidades.

Os profissionais de saúde e dentre eles os enfermeiros que assistem a pessoas com tuberculose, não devem ser culpabilizados por uma dinâmica assistencial que privilegia a norma técnica, pois estão empenhados em restabelecer a saúde dos doentes. Esta é a tradição dos serviços de saúde, cuidar de modo impessoal. Cabe a nós profissionais de saúde superar a natureza impessoal do cotidiano e prover o cuidado que liberta o outro para seu ser mais próprio, o cuidado autêntico. 


\section{REFERÊNCIAS}

1. Nagavci BL et al. Inequalities in tuberculosis mortality: long-term trends in 11 European countries. The International Journal of Tuberculosis and Lung Disease. vol. 20, n. 5, p. 574-581, 2016.

2. Acosta Lisiane Morelia Weide, Bassanesi Sérgio Luiz. The Porto Alegre paradox: social determinants and tuberculosis incidence. Rev. bras. epidemiol. [Internet]. 2014 [cited 2019 June 10] ; 17( Suppl 2 ): 88-101. Available from: http://www.scielo.br/scielo.php?script=sci_arttext\&pi$d=S 1415-790 \times 2014000600088 \&$ ing $=e n$

3. Freitas Wiviane Maria Torres de Matos, Santos Cricia Cristina dos, Silva Monique Mesquita, Rocha Gabriela Amim da. Perfil clinico-epidemiológico de pacientes portadores de tuberculose atendidos em uma unidade municipal de saúde de Belém, Estado do Pará, Brasil. Rev Pan-Amaz Saude [Internet]. 2016 Jun [citado 2019 Jun 10]; 7( 2 ): 45-50. Disponivel em: http://scielo.iec.gov.br/scielo.php?script=sci_arttextEpi$d=S 2176-62232016000200045 \varepsilon \operatorname{lng}=p t$.

4. Ministério da Saúde (BR). Secretaria de Vigilância em Saúde. Boletim Epidemiológico. Implantação do Plano Nacional pelo Fim da Tuberculose como Problema de Saúde Pública no Brasil: primeiros passos rumo ao alcance das metas. v.9, n.1l, mar, 2018. Disponivel em:<http://portalarquivos2.saude.gov.br/images/pdf/2018/marco/26/2018-009.pdf>. Acesso em 14 Jun 2018>.

5. World Health Organization. Global tuberculosis report 2018 [Internet]. Geneva, 2018. Disponivel em:<https://apps.who.int/iris/bitstream/handle/10665/274453/9789241565646-eng.pdf>.Acessado em 12 Jan 2019.

6. Wysocki Anneliese Domingues, Ponce Maria Amélia Zanon, Brunello Maria Eugênia Firmino, Beraldo Aline Ale, Vendramini Silvia Helena Figueiredo, Scatena Lúcia Marina et al. Atenção Primária à Saúde e tuberculose: avaliação dos serviços. Rev. bras. epidemiol. [Internet]. 2017 Mar [cited 2019 June 10] ; 20(1): 161-175. Available from: http://www.scielo.br/scielo.php?script=sci_arttextEpid=S1415-790X20170001001618lng=en.

7. Orfão NH, Andrade RLP, Beraldo AA, Brunello MEF, Scatena LM, Villa TCS. Adesão terapêutica ao tratamento da tuberculose em um municipio do Estado de Sao Paulo. Cienc Cuid Saude. [Internet]. 2015 [citado 2016 Jun 06]; Out/Dez; 14(4):1453-1461. Disponivel em: http://ojs.uem.br/ojs/ index.php/CiencCuidSaude/article/view/25093.

8. Tadesse, Takele, Demissie Meaza, Berhane Yemane, Kebede Yigzaw, Abebe Markos. Long distance travelling and financial burdens discourage tuberculosis DOTs treatment initiation and compliance in Ethiopia: a qualitative study. BMC PublicHealth [Internet]. 2013 [citado em 04 Jun 2016]. Disponivel em: http://bmcpublichealth.biomedcentral.com/articles/10.1186/1471-2458-13-424.

9. Oliveira LCS, Nogueira JA, Sá LD, Palha PF. A discursividade do sujeito sobre sentimentos associados ao enfrentamento da tuberculose. Rev. Eletr. Enf. [Internet]. 2015 jan./mar.:17(1):12-20. Disponivel em: http://dx. doi.org/10.5216/ree.v17il.24523. Acesso em 05 ago 2015.

10. Herrero MB, Ramos S, Arrossi S. Determinants of nonadherence to tuberculosis treatment in Argentina: barriers related to access to treatment. Rev Bras Epidemiol. 2015 Abr/Jun; 18(2): 287-298.

11. Silva CCAV, Andrade MS, Cardoso MD. Fatores associados ao abandono do tratamento de tuberculose em individuos acompanhados em unidades de saúde de referência na cidade do Recife, Estado de Pernambuco, Brasil, entre 2005 e 2010. Epidemiol Serv Saúde. [Internet]. 2013 [citado 2017 Feb 27]; 22(1):77-85. Disponivel em: http://scielo.iec.pa.gov.br/pdf/ ess/v22nl/v22nla08.pdf.

12. Silva PF, Moura GS, Caldas AJM. Fatores associados ao abandono do tratamento da tuberculose pulmonar no Maranhão, Brasil, no periodo de 2001 a 2010. Cad Saúde Pública [Internet]. 2014 [citado 2017 Jan
15]; 30(8):1745-54. Disponivel em: http://www.scielo.br/scielo.php?pid=S0102-311X2014000801745Escript=sci_arttext.

13. Bertolozzi MR, Takahashi RF, Hino P, Litvoc M, França FOS. O controle da tuberculose: um desafio para a saúde pública. Rev Med (São Paulo) [Internet]. 2014 [cited 2015 Jul 11]; 93(2):83-9. Disponivel em: http://www. revistas.usp.br/revistadc/article/view/97330.

14. Mendes EV. O cuidado das condições crônicas na atenção primária à saúde: o imperativo da consolidação da estratégia da saúde da família. Brasilia, DF: Organização Pan-Americana da Saúde; 2012. 512p.

15. Polit DF, Beck CT, Hungler BP. Fundamentos de pesquisa em enfermagem. Métodos, avaliação e utilização. 6. ed. São Paulo: Artmed; 2011.

16. Oliveira MFV, Carraro TE. Cuidado em Heidegger: uma possibilidade ontológica para enfermagem. Rev Bras Enferm, [Internet]. 2011 [citado em 05 Jan 2017];64(2):376-80. Disponivel em: http://www.scielo.br/pdf/ reben/v64n2/a25v64n2.pdf.

17. Heidegger M. Ser e tempo. $5^{\circ}$ ed. trad. rev. Márcia de Sá Cavalcante. Petrópolis: Vozes, 2011.

18. Touso MM, Popolin MP. Crispim JA, Freitas IM de, Rodrigues LBB, Yamamura M et al. Estigma social e as familias de doentes com tuberculose: um estudo a partir das análises de agrupamento e de correspondência múltipla. Ciênc. saúde coletiva [Internet]. 2014 Nov [citado em Jun 2018]; 19( 11 ): 4577-4586. Disponivel em: http://www.scielo.br/scielo.php?scrip$\mathrm{t}=$ sci_arttextEpid=S1413-81232014001104577\&lng=en.

19. Oliveira CBB de, Lima MCRA D'Auria de, Faria MF, Ravanholi GM, Lopes LM, Souza KM de et al. Experiências de adoecimento por condições crônicas transmissiveis: revisão integrativa da literatura. Saúde soc. [Internet]. 2017 June [citado em Jun 2018] : 26( 2 ): 510-520. Disponivel em: http://www.scielo.br/scielo.php?script=sci_arttextEpi$d=$ S0104-12902017000200510\&lng=en.

20. Santos MNA, Sá AM M. Viver com tuberculose em prisões: o desafio de curar-se. Texto contexto - enferm. [Internet]. 2014 Dec [citado em Jun 2018]; 23( 4 ): 854-861. Disponivel em: http://www.scielo.br/scielo.php?script=sci_arttextEpid=S0104-07072014000400854Elng=en.

21. Chirinos N C, Meirelles BHS, Bousfield ABS. Representações sociais das pessoas com tuberculose sobre o abandono do tratamento. Rev. Gaúcha Enferm. [Internet]. 2015 [citado em Jun 20186] ; 36( spe ): 207214. Disponivel em: http://www.scielo.br/scielo.php?script=sci_arttextEpid=S1983-14472015000500207\&lng=en.

22. Silva AKVL, Junior DNS, Silva YR, Nascimento EGC. Fatores associados ao tratamento da tuberculose na perspectiva do usuário, família e assistência. Com. Ciências Saúde. 2014; 25(3/4):275-290. Disponivel em: http:// bvsms.saude.gov.br/bvs/periodicos/ccs_artigos/2014_fatores_associados_tratamento.pdf. Acesso em 14 nov 2016.

23. Souza KMJS, Lenilde DS, Filomena EPAi, Rodrigo PFQ, Catiucia AS, Pedro FP. Discursos sobre a tuberculose: estigmas e consequências para o sujeito adoecido. Rev enferm UERJ, Rio de Janeiro, 2015 jul/ago; 23(4):475-80. Disponivel em:< http://www.facenf.uerj.br/v23n4/v23n4aO7. pdf.

24. Farias SNP, Medeiros CRS, Paz EPA, Lobo AJS, Ghelman LG.Integralidade no cuidado: estudo da qualidade de vida dos usuários com tuberculose. Esc. Anna Nery [Internet]. 2013 [citado em Jan 2019];17(4):749-54. Disponivel em: http://www.scielo.br/pdf/ean/v17n4/1414-8145-ean-17-04-0749. pdf. 